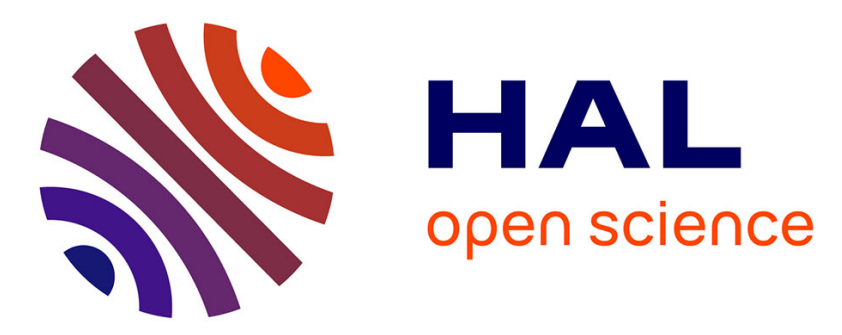

\title{
La difficile territorialisation des stratégies nationales de gestion des risques côtiers en France
}

\author{
Lucile Mineo-Kleiner, Céline Perherin, Catherine Meur-Ferec, Camille Noûs
}

\section{To cite this version:}

Lucile Mineo-Kleiner, Céline Perherin, Catherine Meur-Ferec, Camille Noûs. La difficile territorialisation des stratégies nationales de gestion des risques côtiers en France. Annales de géographie, 2021, $\mathrm{N}^{\circ} 738$ (2), pp.50-76. 10.3917/ag.738.0050 . hal-03434356

\section{HAL Id: hal-03434356 https://hal.science/hal-03434356}

Submitted on 18 Nov 2021

HAL is a multi-disciplinary open access archive for the deposit and dissemination of scientific research documents, whether they are published or not. The documents may come from teaching and research institutions in France or abroad, or from public or private research centers.
L'archive ouverte pluridisciplinaire HAL, est destinée au dépôt et à la diffusion de documents scientifiques de niveau recherche, publiés ou non, émanant des établissements d'enseignement et de recherche français ou étrangers, des laboratoires publics ou privés. 


\title{
La difficile territorialisation des stratégies nationales de gestion des risques côtiers en France
}

\author{
The enforcement issues for national policy on coastal \\ risks management in France
}

Lucile Mineo-Kleiner

Docteure en géographie, Université de Bretagne occidentale, Laboratoire Littoral Environnement Télédétection Géographie (LETG) - UMR 6554 CNRS.

\section{Céline Perherin}

Ingénieure des travaux publics de l'État, docteure en géographie, Cerema, chercheure associée au laboratoire LETG-UMR 6554 CNRS.

\section{Catherine Meur-Ferec}

Professeure de géographie, Université de Bretagne occidentale, Laboratoire LETG - UMR 6554 CNRS.

\section{Camille Noûs}

Université de Bretagne occidentale, laboratoire Cogitamus.

Résumé Depuis une quarantaine d'années, l'État a développé un arsenal d'outils et de stratégies sur la gestion des risques côtiers. Néanmoins, leur mise en œuvre sur les territoires reste délicate. Un décalage de positions entre l'État et les collectivités locales s'observe et témoigne d'une territorialisation difficile de cette politique. Nous proposons ici une explication aux difficultés rencontrées à travers une analyse portant sur les échelles d'action géographique et l'héritage de la décentralisation.

Abstract For forty years, the French government has developed a legal arsenal and several strategies concerning coastal risks management. Nevertheless, their enforcement at local level remains an issue. A gap between the positions of the French government and local authorities is observed and is indicative of the difficulty encountered for enforcement of this policy. This paper proposes an explanation for these difficulties, based on our the Authors'analysis of scales of operation and decentralization heritage.

Mots-clés gestion des risques côtiers, gouvernance, analyse de politique publique, décentralisation, échelle locale/centrale

Keywords coastal risks management, governance, public policy analysis, decentralization, local/national scale

\section{Introduction}

Les submersions marines de grande ampleur dans les Pays de la Loire et en Poitou-Charentes lors de la tempête Xynthia du 28 février 2010 ont entraîné une 
véritable prise de conscience du retard accumulé dans l'application des politiques de prévention des risques littoraux. L'État et les collectivités ne s'étaient jusqu'à présent que peu engagés dans cette voie (Meur-Ferec, 2006 ; Deboudt, 2010). Suite à cet évènement, l'État a mis en œuvre des outils réglementaires et normatifs existants, d'autres ont été créés. La réglementation sur les ouvrages de protection contre les inondations s'est renforcée. Les programmes d'actions et de prévention des inondations (PAPI) ont été élargis à la submersion marine. Enfin, l'adoption de la stratégie nationale de gestion intégrée du trait de côte a largement été nourrie des enseignements tirés de cet événement.

Cependant, malgré un arsenal d'outils et de stratégies développés par l'État, leur application sur les territoires reste délicate. En particulier, depuis 2011, la mise en œuvre des plans de prévention des risques littoraux (PPRL) a fait émerger de nombreuses situations de contestations locales.

À travers cet article, nous proposons une explication aux difficultés rencontrées lors de la territorialisation de la politique de gestion des risques côtiers selon une analyse portant sur les échelles d'action géographique et l'héritage de la décentralisation. L'État est resté très présent dans ce domaine, alors même que plusieurs phases de décentralisation se sont succédé. Notre hypothèse est que nous traversons une période charnière dans la politique de gestion des risques côtiers, 10 ans après la tempête Xynthia. Les tensions existantes lors de la territorialisation des politiques de gestion des risques côtiers seraient révélatrices de l'évolution des rôles de l'État et des collectivités, marquées par l'affirmation de politiques nationales, de fortes évolutions de compétences, d'outils et de réglementations, à l'origine d'un brouillage des repères. Le positionnement de l'État et des collectivités serait ainsi aujourd'hui en quête de stabilisation.

Cet article de synthèse écrit à trois mains repose sur des résultats issus de deux thèses de doctorats de géographie sur la gestion des risques côtiers de Lucile Mineo-Kleiner (Mineo-Kleiner, 2017) et de Céline Perherin (Perherin, 2017) et sur l'expérience de recherche de Catherine Meur-Ferec qui les a dirigées (Meur-Ferec, 2006). Le tableau suivant (Tab. 1) synthétise des éléments méthodologiques concernant les résultats utilisés dans le cadre de cet article.

Dans un premier temps, nous présenterons brièvement les compétences respectives de l'État et des collectivités en matière de gestion des risques côtiers. La mise en perspective historique des relations entre ces deux acteurs publics, réalisée dans un second temps, nous permettra dans une troisième partie de comprendre les difficultés auxquelles la territorialisation de la politique de prévention des risques côtiers fait face. Afin de faciliter la lecture de cet article, nous avons recensé l'ensemble des acronymes utilisés dans le tableau ci-dessous (Tab. 2). 
Tab. 1 Méthode d'acquisition des résultats mobilisés dans le cadre de cet article. Collection method of results presented in this paper.

\begin{tabular}{|c|c|c|c|}
\hline & & Thèse de L. Mineo-Kleiner & Thèse de C. Perherin \\
\hline Type & d'enquête & Entretiens s & mi-directifs \\
\hline & $\begin{array}{l}\text { Services de l'État : } \\
\text { DREAL et DDTM }\end{array}$ & 12 & 9 \\
\hline $\begin{array}{l}\text { d'enquêtés } \\
\text { par catégorie }\end{array}$ & $\begin{array}{l}\text { Collectivités } \\
\text { locales (élus et } \\
\text { services } \\
\text { techniques) }\end{array}$ & 11 & 21 \\
\hline Sites étudiés & département) & $\begin{array}{l}\text { Hyères }(83) \\
\text { Vias (34) } \\
\text { Ault (80) } \\
\text { Lacanau et La } \\
\text { Teste-de-Buch (33) } \\
\text { Labenne (40) }\end{array}$ & $\begin{array}{l}\text { Marquenterre - Baie de } \\
\text { Somme (80), } \\
\text { Les pays Bigouden et } \\
\text { Fouesnantais (29), } \\
\text { La Presqu'île de Guérande-- } \\
\text { Saint-Nazaire (44), } \\
\text { L'île de Ré (17). }\end{array}$ \\
\hline Périodes d'en & quête & 2014 & 2016 \\
\hline
\end{tabular}

Tab. 2 Liste des acronymes.

List of acronyms.

\begin{tabular}{|c|c|}
\hline Acronymes & Signification \\
\hline CGEDD & Conseil général de l'environnement et du développement durable \\
\hline DCE & Directive-cadre sur l'eau \\
\hline DDTM & Direction départementale des territoires et de la mer \\
\hline DICRM & Document d'information communal sur les risques majeurs \\
\hline DREAL & Direction régionale de l'environnement, de l'aménagement et du logement \\
\hline GEMAPI & Gestion des milieux aquatiques et protection contre les inondations \\
\hline Loi LENE & \begin{tabular}{|l} 
Loi portant engagement national pour l'environnement \\
\end{tabular} \\
\hline Loi MAPTAM & $\begin{array}{l}\text { Loi de modernisation de l'action publique territoriale et d'affirmation des } \\
\text { métropoles }\end{array}$ \\
\hline Loi NOTRe & Loi portant sur la nouvelle organisation territoriale de la République \\
\hline MATE & Ministère de l'aménagement, du territoire et de l'environnement \\
\hline MEDD & Ministère de l'écologie et du développement durable \\
\hline MEEM & Ministère de l'environnement, de l'énergie et de la mer \\
\hline METL & Ministère de l'équipement, des transports et du logement \\
\hline MLHD & Ministère du logement et de l'habitat durable \\
\hline ORSEC & Organisation de la réponse de sécurité civile \\
\hline PAPI & Programmes d'actions et de prévention des inondations \\
\hline PCS & Plan communal de sauvegarde \\
\hline PER & Plan d'exposition aux risques \\
\hline PLU & Plan local d'urbanisme \\
\hline PPR/PPRN/PPRL & $\begin{array}{l}\text { Plan de prévention des risques, plan de prévention des risques naturels, } \\
\text { plan de prévention des risques littoraux }\end{array}$ \\
\hline $\begin{array}{l}\text { Régime Cat } \\
\text { Nat }\end{array}$ & Régime de catastrophes naturelles \\
\hline SCoT & Schéma de cohérence territorial \\
\hline
\end{tabular}




\section{Un système de gouvernance structuré autour de deux catégories d'acteurs publics}

\subsection{Un État très présent dans la prévention des risques côtiers}

En tant que membre de l'Union européenne, la France est tenue d'adopter les mesures nécessaires pour l'application des lois européennes. Deux directives interviennent dans la gestion des risques côtiers : la directive-cadre sur l'eau ${ }^{1}$ (DCE) et la directive inondation ${ }^{2}$. La DCE n'intervient que de façon limitée et indirecte sur les inondations. La directive inondation incite à mettre en place une vision stratégique du risque. Elle a été retranscrite dans la loi portant engagement nationale pour l'environnement (LENE) du 12 juillet 2010. L'Union Européenne a également financé des projets de recherche tels que le programme Eurosion. Aujourd'hui l'Union européenne instaure un cadre dans lequel les États membres évoluent. Néanmoins, concernant la gestion des risques côtiers, ce cadre, en cours de construction, est lacunaire. Il concerne des compétences non exclusives de l'Union Européenne et doit donc répondre au principe de subsidiarité. Dans la suite de cet article, nous nous intéresserons principalement à la relation entre l'État et les collectivités.

Selon ses grands principes, l'État est garant de l'intérêt général et de l'égalité de traitement des citoyens par opposition aux intérêts privés (Rosanvallon, 1990). Cette conception de l'intérêt général, ancrée dans la notion d'État providence (Ewald, 1986), marque fortement les politiques publiques à laquelle les politiques de gestion des risques littoraux ne font pas exception.

L'État, assure un rôle de "guide ». Il suit une approche thématique sur des sujets qui s'appliquent à l'ensemble du territoire national et sur le long terme. Ces échelles spatiales et temporelles sont le propre de l'État. Par exemple, l'érosion et la submersion marine sont abordées à travers des politiques différentes. Pour chacune d'entre elles, l'État énonce sa doctrine à l'aide une stratégie nationale, influencée par les réflexions menées au niveau européen.

Ainsi, en 2012, l'État adopte la stratégie nationale de gestion intégrée du trait de côte. Elle prône une gestion territoriale des risques impliquant toutes les parties prenantes, à une échelle spatiale adaptée et à des échelles de temps permettant d'anticiper les phénomènes naturels. Elle propose d'envisager une stratégie locale de gestion, dont la mise en œuvre repose sur un portage par les collectivités.

Puis, en 2014, l'État adopte la stratégie nationale de gestion des risques d'inondation. Elle permet l'application de la directive européenne inondation ${ }^{3}$. Elle définit trois priorités à l'échelle nationale : renforcer la sécurité des personnes,

1 Directive-cadre sur l'eau 2000/60/CE adoptée le 23 octobre 2000.

2 Directive européenne 2007/60/CE relative à l'évaluation et la gestion des risques d'inondation, adoptée le 23 octobre 2007.

3 Directive européenne 2007/60/CE relative à l'évaluation et à la gestion du risque inondation. 
réduire le coût des dommages et raccourcir les délais de retour à la normale après un sinistre. Son financement repose sur le principe de solidarité nationale.

Afin de mener ses grandes orientations stratégiques, l'État agit dans plusieurs champs d'intervention et met en place des outils spécifiques.

- L'État informe. Il a un devoir de "porter à connaissance ${ }^{4}$ » : de transmettre aux communes et leur groupement toutes informations utiles pour l'exercice de leur compétence d'urbanisme. Le public est informé des risques notamment à travers le dossier départemental des risques majeurs.

- L'État alerte : la surveillance, la prévision et l'élaboration de la vigilance sont de son ressort. Depuis la tempête Xynthia, le dispositif de vigilance «vague submersion » de Météo France a été créé. L'ensemble des dispositifs reposent sur l'action conjointe de Météo-France, du Shom, de la Direction générale de la prévention et des risques et de la Direction générale de la sécurité civile et gestion des crises pour la mise en œuvre opérationnelle. L'alerte transite par le préfet jusqu'aux communes. La gestion de crise est organisée à un niveau supra-communal par les plans ORSEC (Organisation de la réponse de sécurité civile).

- L'État joue le rôle de réassureur principal dans l'indemnisation de catastrophes naturelles. En effet, les risques naturels, considérés comme non assurables par les contrats d'assurance de dommages aux biens, tels que les mouvements de terrain, les inondations et les submersions, sont couverts par le régime public d'indemnisation des catastrophes naturelles ${ }^{5}$ (régime Cat Nat). Ce système repose sur le principe de solidarité nationale : d'une part, une garantie supplémentaire contre les effets des catastrophes naturelles est obligatoirement incluse dans tout contrat d'assurance de dommages aux biens ; d'autre part, tous les assurés participent de la même façon au financement du régime Cat Nat (prélèvement d'une «surprime» sur leurs cotisations), indépendamment de leur exposition aux aléas (Cazeaux et al., 2019). Dans le cadre du régime Cat Nat, l'État détient et apporte une garantie financière à la Caisse centrale de réassurance à laquelle presque toutes les compagnies d'assurance souscrivent (André, 2013). Ainsi, l'accent a été mis sur la réparation des dommages (Dubois-Maury, 2002) : « rares sont les pays où la couverture [...] est aussi systématique » (Ledoux, 2006, p. 175). Néanmoins, témoignant de l'approche cloisonnée de l'État, l'érosion des côtes basses sableuses ne fait pas partie des risques éligibles au régime Cat Nat.

- L'État veille à limiter l'urbanisation en zone à risque. Il contrôle les autorisations d'urbanisme et instruit les PPR, outil central de la prévention des risques. Dans ce cadre, il mène les études, organise la concertation, réalise les cartes réglementaires. La prise en compte des risques dans l'aménagement garantit la sécurité des biens et des personnes et assure la pérennité du régime Cat Nat. En effet, ce dernier pourrait être mis en péril par la multiplication des constructions

4 Articles L 121-1 et R 121-1 du Code de l'Urbanisme.

5 Créé par la loi n 82-600 du 13 juillet 1982 relative à l'indemnisation des victimes des catastrophes naturelles. 
en zones exposées aux aléas, poussée par les intérêts particuliers (Barraqué, 1994), ainsi que par l'exposition de nouveaux espaces en raison de l'augmentation du niveau marin moyen liée aux changements climatiques. En outre, afin d'inciter la mise en œuvre des PPR, l'État a décidé de renforcer le lien entre indemnisation et prévention (Meur-Ferec, 2006). Le dispositif mis en place en 2002 prévoit une modulation de la franchise de base des assurances dans les communes où un PPR n'a pas été prescrit, ou si un PPR n'a pas fait l'objet d'une approbation dans le délai de 4 ans suivant sa date de prescription. Les franchises, payées par les particuliers augmentent en fonction du nombre d'arrêtés de catastrophes naturelles dont la commune a fait l'objet depuis 1995 (loi Barnier), pour une même nature de péril. Cette responsabilisation des particuliers, et donc des élus locaux, a entraîné une très nette augmentation du nombre de PPR en cours d'élaboration. Par ailleurs, depuis la stratégie nationale de gestion intégrée du trait de côte, l'État tente d'amorcer les réflexions autour de la relocalisation des biens exposés aux aléas.

- Enfin, l'État décide des projets qui bénéficieront de financement. Il est le financeur de certaines mesures préventives par le Fonds de prévention des risques naturels majeurs (dit fonds Barnier), alimenté par une part de la surprime Cat Nat (Cazeaux et al., 2019). À l'aide de dispositifs de contractualisation, il finance les projets locaux cohérents avec ses stratégies. Il s'agit par exemple des appels à projets de PAPI. Les PAPI ont pour objectif de fournir un cadre pour l'accompagnement des projets de prévention portés par les collectivités territoriales. . Ces programmes pluriannuels sont tenus d'inclure des actions sur l'ensemble des axes de la prévention des inondations. Ils doivent reposer sur une approche globale à une échelle adaptée.

Les actions de prévention des risques sont coordonnées par le ministère chargé de l'environnement (actuellement ministère de la Transition écologique). La prévention des risques incombe à la Direction Générale de la Prévention des Risques. La Direction Générale de l'Aménagement du Logement et de la Nature se charge également des questions relatives à la gestion du trait de côte, et entre-autre, de l'urbanisme. La gestion de crise quant à elle, relève principalement du Ministère de l'Intérieur. L'action du ministère s'appuie sur ses services déconcentrés en région (DREAL) et en département (DDTM).

Ainsi, l'État occupe traditionnellement une place centrale dans la gestion des risques naturels (Meur-Ferec et Morel, 2004; Deboudt, 2010), mais également dans la gestion du littoral, considéré comme un patrimoine de la Nation (Merckelbagh, 2009). Il reste le garant des intérêts nationaux, des valeurs fondamentales. Il détient un rôle d'orientation des politiques et un rôle d'impulsion des dynamiques locales. Il garde également un rôle de contrôle. Suivant un objectif d'égalité de traitement des citoyens sur l'ensemble du territoire français, les grands principes de l'État tendent à être monothématiques et déterritorialisés. De leur côté, les collectivités veillent au bon fonctionnement de leur territoire tout en appliquant les grands principes fixés par l'État. 


\subsection{Des collectivités centrées sur leur territoire}

Les collectivités territoriales ${ }^{6}$ sont chargées du développement économique et social de leur territoire. À la différence des services de l'État, elles intègrent une multitude de thématiques. Elles exercent une série de compétences dans la gestion des risques littoraux.

- Les collectivités définissent les orientations d'aménagement du territoire à travers le schéma de cohérence territorial (SCoT) et le plan local d'urbanisme (PLU). Le maire délivre les permis de construire dans le respect des textes réglementaires et sous le contrôle de légalité de l'État. En outre, selon l'article R. 111-2 du Code de l'Urbanisme, si le maire a connaissance d'un risque sur une parcelle, il peut et doit refuser de délivrer un permis de construire, même si ce risque ne figure pas dans les documents d'urbanisme ou le PPR.

- En tant qu'agent de l'État, le maire assure aussi la sécurité des habitants et détient le pouvoir de police. Il est responsable du maintien de l'ordre public sur sa commune. Ainsi, il a l'obligation d'intervenir si la sécurité des habitants est en jeu. Dans le cas d'un bâtiment menacé par des risques naturels, le maire peut établir un arrêté de péril imminent.

- Le maire est également chargé d'informer la population sur les risques auxquels elle est exposée, de diffuser l'alerte et d'organiser les secours à l'échelle communale. Deux outils principaux permettent d'assurer ces missions. Il s'agit du plan communal de sauvegarde (PCS) où sont définies les mesures de sauvegarde et de protection des personnes et les moyens disponibles en cas de crise. Le second outil est le Document d'information communal sur les risques majeurs (DICRIM). Il expose entre autres les caractéristiques des risques et les événements historiques connus. Ces documents sont obligatoires, notamment lorsque la commune est concernée par un PPR.

- Les collectivités détiennent depuis peu la compétence pour la prévention des inondations ${ }^{7}$. À travers la compétence GEMAPI (gestion des milieux aquatiques et protection contre les inondations), elles définissent leurs systèmes d'endiguement, et répondent aux obligations les concernant (entretien, gestion, surveillance, etc.). À ce titre, elles sont autorisées à prélever une taxe.

Pour assurer plusieurs de ces compétences, les collectivités sollicitent l'appui et le financement de l'État, par exemple au travers des labellisations des PAPI.

Les collectivités territoriales intègrent la gestion des risques à l'ensemble des thématiques au sein d'une approche territoriale nécessairement intégrée. Le maire occupe une place particulière : il est à la fois agent de l'État, et en ce sens garant de l'intérêt général, et élu local, responsable du développement du territoire communal et de la défense de ses administrés.

6 Dans la suite de cet article, nous désignons par collectivités territoriales locales les communes et leurs groupements.

7 La loi $n^{\circ}$ 2014-58 du 27 janvier 2014 de modernisation de l'action publique territoriale et d'affirmation des métropoles, dite loi MAPTAM crée la compétence GEMAPI. 
Ainsi, pour synthétiser, la répartition des compétences entre l'État et les collectivités dans la gestion des risques côtiers est représentée par la figure 1. Il s'agit d'une version réactualisée des travaux de C. Meur-Ferec et Y. Rabuteau (2014).
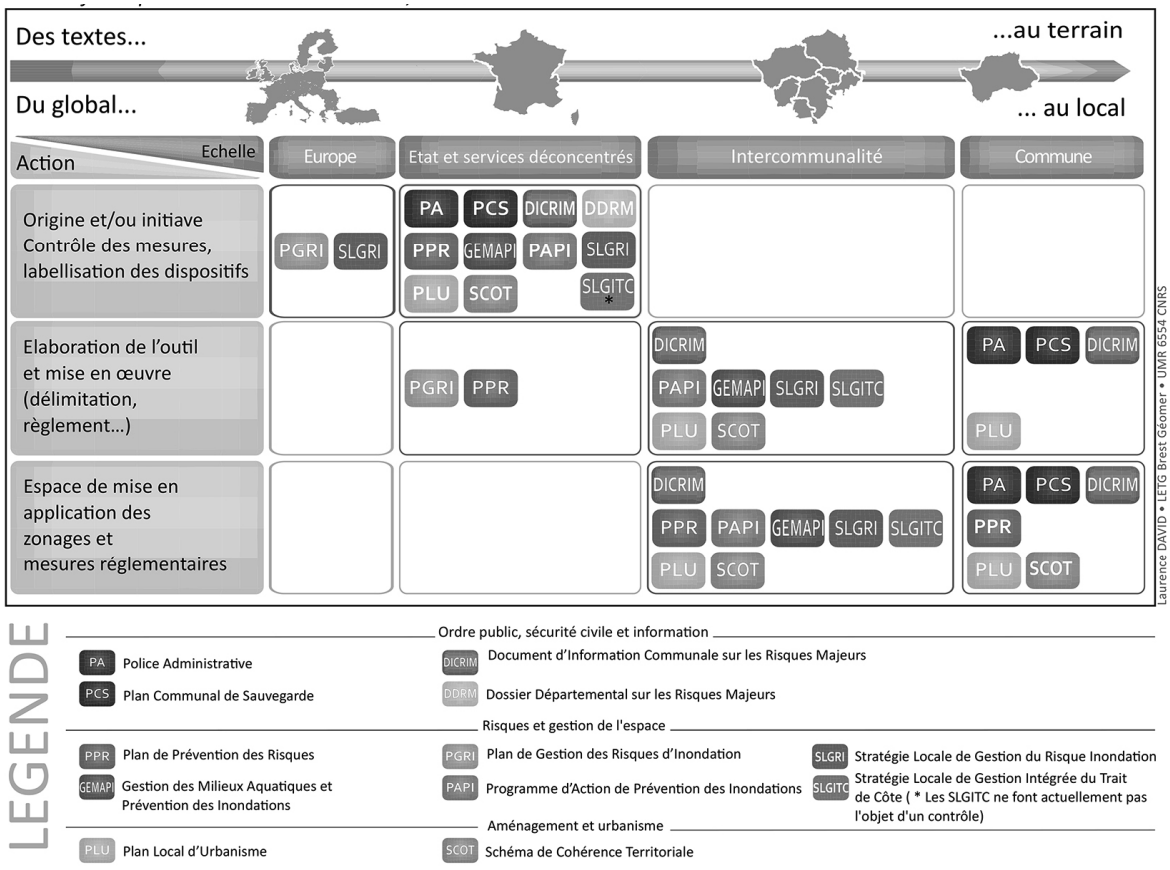

Fig. 1 Matrice multi-scalaire des principaux instruments de gestion des risques côtiers (modifiée d'après Meur-Ferec et Rabuteau, 2014).

Matrix of the main coastal risk management tools (modified from Meur-Ferec and Rabuteau, 2014)

Cette répartition actuelle des compétences est l'héritage d'une évolution historique de partage des pouvoirs entre ces deux principaux acteurs, somme toute assez récente.

\section{L'évolution historique de la gouvernance à travers les relations État-collectivités}

\subsection{Historiquement, un État expert hégémonique}

Le rôle de l'État dans la politique de prévention des risques côtiers s'explique par la place historique de défenseur de l'intérêt général, qui lui confère un pouvoir symbolique. Néanmoins l'héritage de l'État-providence est aussi un 
« fractionnement de l'appareil d'État, [...un] cloisonnement administratif, [...et une] emprise techniciste d'expertises spécialisées » (Thoenig et Duran, 1996, p. 591).

Le besoin d'expertise conduit l'État à se doter de "grands corps » de spécialistes (Lascoumes, 2002 ; Decrop, 2013). L'État fonctionne alors sur un modèle dit «décisionniste » dans lequel une solution « rationnelle » peut répondre à chaque problème (Habermas, 1973). À cette époque, la totalité de l'expertise scientifique est détenue par l'État. La politique de prévention des risques côtiers est alors entièrement centrée sur la maitrise de l'aléa par des ouvrages de défense contre la mer, construits suite aux dégâts provoqués par les tempêtes.

Il faut attendre les années 1980 pour que cette politique commence à s'infléchir et que de nouveaux acteurs fassent leur apparition. La France définit alors la prise en compte des risques dans l'aménagement du territoire comme un axe fort de la prévention des risques naturels avec l'indemnisation des catastrophes naturelles. L'objectif évolue : il s'agit de limiter l'implantation de nouveaux enjeux en zone à risque et de réduire la vulnérabilité de ceux déjà présents, par des ouvrages de défense ou par des mesures sur les constructions. En imposant des restrictions à l'aménagement local, l'État s'inscrit dans son rôle traditionnel de défense de l'intérêt général. La réalisation des plans d'exposition aux risques (PER), réglementant l'urbanisme, se base sur l'expertise traditionnelle de l'État portée par un ou plusieurs experts, qui définit un "aléa non négociable » pour tout acteur. L'approche uniquement «scientifique » prédomine.

\subsection{La montée en compétences des collectivités dans le cadre de la décentralisation}

Une volonté de démocratisation et de participation des citoyens conduit à une recherche de "démocratie locale » (Paoletti, 2007). Le constat partagé que l'État ne peut plus décider seul s'accompagne de plusieurs phases de décentralisation depuis le début des années 1980. La décentralisation se traduit par un transfert progressif de compétences de l'État vers les collectivités territoriales. Elle s'est réalisée en plusieurs phases marquées par des évolutions législatives : l'acte I, en 1982 avec les lois Defferre ${ }^{8}$, l'acte II, marqué par une révision constitutionnelle en $2003^{9}$ et la loi relative aux libertés et responsabilités locales de 2004, et l'acte III en 2015 principalement marqué par la « loi NOTRe ${ }^{10}$. L'État conserve la responsabilité de la définition et de la conduite de la politique économique et sociale, mais les régions, les départements et les communes concourent avec lui à

8 Loi n 82-213 du 2 mars 1982 relative aux droits et libertés des communes, des départements et des régions, loi n 83-8 du 7 janvier 1983 relative à la répartition des compétences entre les communes, les départements, les régions et l'État et loi n 83-663 du 22 juillet 1983 qui complète la loi du 7 janvier 1983.

9 Loi constitutionnelle $\mathrm{n}^{\circ}$ 2003-276 du 28 mars 2003, relative à l'organisation décentralisée de la République

10 Loi n 2015-991 du 7 août 2015 portant sur la nouvelle organisation territoriale de la République, 
l'aménagement du territoire, au développement économique et à la protection de l'environnement. Ainsi, depuis la loi du 7 janvier 1983, l'urbanisme et le droit des sols deviennent une compétence communale : les plans d'occupation des sols (devenus plans locaux d'urbanisme) sont élaborés et approuvés par les communes. L'État garde cependant la main sur la prise en compte du risque dans l'aménagement du territoire (Mazé et Meur-Ferec, 2017). Un second tournant majeur en termes de décentralisation dans la gestion des risques naturels est la prise de compétence de prévention des inondations (compétence GEMAPI) par les collectivités : depuis janvier 2018, elles sont responsables de la gestion de leur système d'endiguement (cf. p. $\underline{56}$ ).

Cette nouvelle répartition des responsabilités entre une multitude d'acteurs locaux, nationaux et européens contribue, sur un territoire convoité comme le littoral, à une logique de « foire d'empoigne »(Meur-Ferec, 2006). L'État entend conserver son rôle d'arbitre face aux pressions, comme en témoigne la loi Littoral de 1986. Pourtant, le rôle protecteur, traditionnel de l'État-providence, n'est plus valorisé. L'État, en mal d'un "pouvoir symbolique extrêmement fort ", détrôné par le principe de subsidiarité, peine à faire valoir son point de vue (Moquay, 2005). Il n'est plus qu'« un acteur parmi d'autres » (Le Galès, 1995). Il perd également son expertise au profit des collectivités (Restier-Melleray, 1990 ; Le Bourhis, 2007) et d'une externalisation des compétences auprès des bureaux d'études (Bezes, 2009). De leur côté, les collectivités revendiquent plus de liberté. La loi Littoral est vivement critiquée localement, les PER n'aboutissent pas. Les acteurs doivent composer ensemble.

Parallèlement, les sciences dites "dures » se heurtent, à la fin des années 1980, à leurs propres limites (accident de Tchernobyl, explosion de la navette Challenger, etc.). Les problèmes environnementaux et la gestion des risques de toute origine conduisent à reconnaitre un nombre croissant de situations d'incertitudes et à une méfiance dans les jugements d'experts (Restier-Melleray, 1990; Lascoumes, 2002). "Le projet quelque peu utopique de conquête de la sécurité, via l'éradication du risque, se trouve dans une impasse et laisse la place à une gestion pragmatique » (Peretti-Watel, 2000). Dans le domaine des risques côtiers, cette incertitude est aussi mise en avant, par les scientifiques eux-mêmes, contribuant à la perte de légitimité des experts, et donc de l'État lors de la caractérisation des aléas.

L'émergence de nombreuses questions environnementales conduit également à des évolutions de la définition de l'action collective plus adaptée aux problèmes complexes (Callon et al., 2001 ; Gaudin, 2002 ; Theys, 2002a). La gouvernance évolue sous l'effet de la recherche de plus de transparence, d'un dialogue avec les experts, d'un accès à l'information, d'une participation élargie aux décisions, et d'une restauration de la confiance. Ainsi, la loi Bouchardeau ${ }^{11}$ de 1983 consacre

11 Loi n 83-360 du 12 juillet 1983 relative à la démocratisation des enquêtes publiques et à la protection de l'environnement. 
la participation du public à toute opération d'aménagement et de planification urbaine susceptible d'affecter l'environnement.

Si le recours aux défenses structurelles est encore privilégié et si les décisions restent principalement fondées sur l'expertise dans les années 1980 et 1990, les années 1990 vont enclencher une nouvelle dynamique dans la gestion des risques littoraux (Deboudt, 2010 ; Meur-Ferec et al., 2013).

L'évolution des PER et des PPR est un bon exemple de la progression de la concertation dans le temps sous l'influence des collectivités. Depuis les années 1980, elles revendiquent en effet une part prédominante dans les décisions ayant trait à l'aménagement du territoire. Elles rejettent massivement le PER, limitant selon elles le développement économique, et sa méthode d'élaboration. Une profonde évolution vers le PPR conduit à ne plus faire uniquement appel à une approche scientifique. Son élaboration s'appuie sur une analyse qualitative et un processus itératif entre production des connaissances et prise de décision, laissant une place importante à la concertation (MATE et METL, 1997 ; Bayet, 2000 ; Hubert et Reliant, 2003). La loi Barnier ${ }^{12}$, consacrant les PPR en 1995, définit clairement les attentes en termes d'acteurs impliqués dans l'élaboration du PPR, de consultation et d'enquête publique. Pour répondre aux critiques récurrentes des élus locaux sur le manque de concertation et aux difficultés d'application par les services, la législation évolue à nouveau. La loi du 30 juillet $2003^{13}$ a ainsi pour objectif de renforcer l'information, la participation du public et la concertation interinstitutionnelle dans le domaine des risques naturels. L'État définit en collaboration avec les élus et les représentants de la société civile les bonnes pratiques ${ }^{14}$ (MEDD, 2006). Ainsi, le schéma ci-dessous (Figure 2) montre que la concertation et l'association avec les collectivités intervient dans toutes les phases d'élaboration d'un PPR. La recherche de la concertation est notamment mise en avant à travers les guides réglementaires tels que le guide «Plan de prévention des risques naturels : le guide de la concertation » (MEDD, 2003).

Au fur et à mesure de la décentralisation et de la mise en place de cette nouvelle organisation, les compétences techniques au sein des services de l'État diminuent (Ledoux, 2006). La réduction des finances publiques ${ }^{15}$ contribue également à une diminution des effectifs des agents de l'État. Cette remise en cause porte sur tous les champs d'expertise, y compris celle de la définition des aléas côtiers, où les services de l'État s'appuient souvent entièrement sur les compétences de prestataires externes (Perherin, 2017). De leur côté, les collectivités font elles

12 Loi n 95-101 du 2 février 1995 relative au renforcement de la protection de l'environnement.

13 Loi n² 2003-699 du 30 juillet 2003 relative à la prévention des risques technologiques et naturels et à la réparation des dommages.

14 Circulaire du 3 juillet 2007 relative à la consultation des acteurs, la concertation avec la population et la consultation des collectivités territoriales dans le cadre des PPRN.

15 La réduction des finances publiques a démarré avec le début de la décentralisation et se traduit plus récemment avec la révision générale des politiques publiques de 2007 et la modernisation de l'action publique de 2012. 


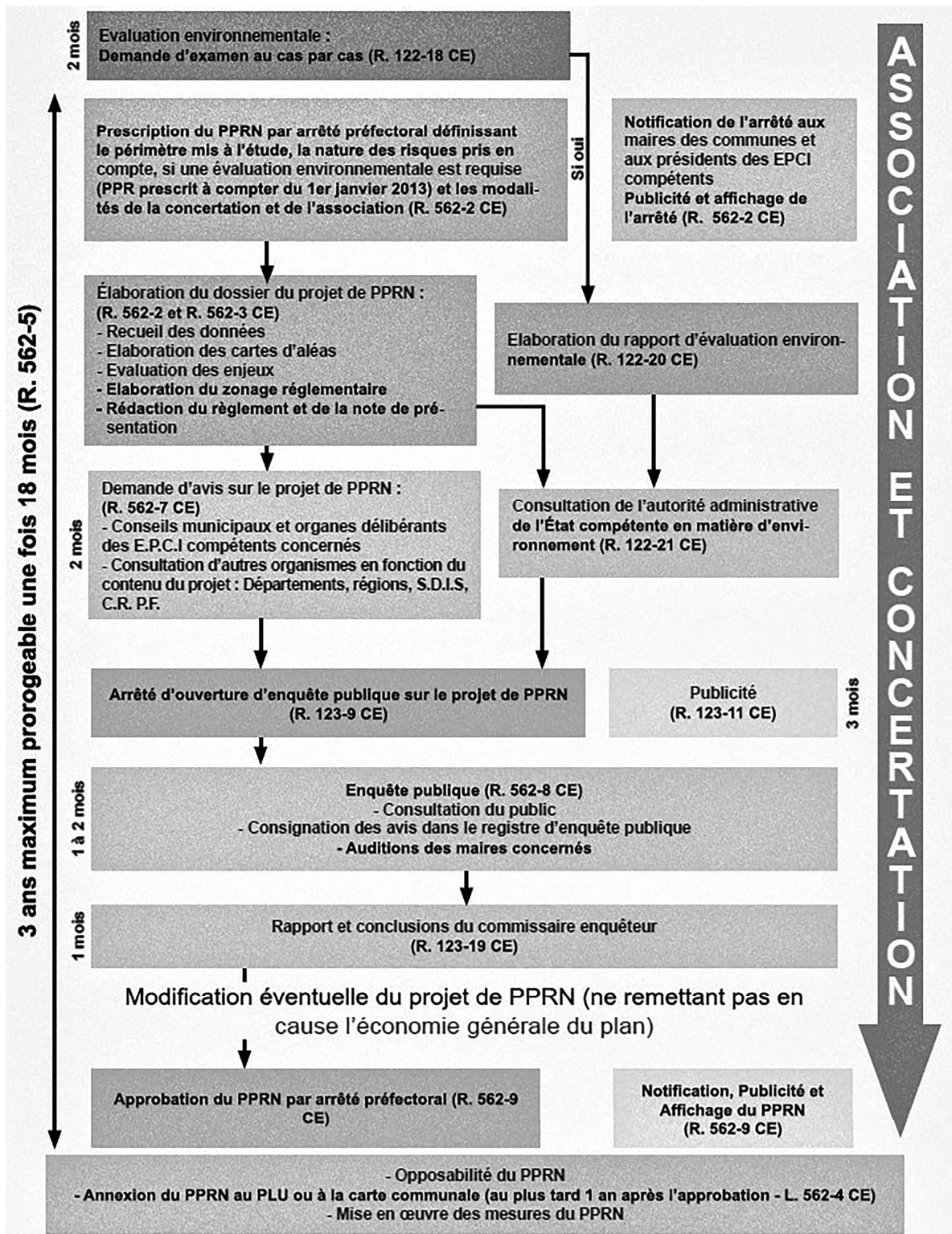

Fig. 2 Schéma détaillé d'élaboration d'un PPR (MEEM et MLHD, 2016). Detailed chart of development of PPR (MEEM and MLHD, 2016). 
aussi appel à des bureaux d'étude lorsqu'elles en ont les moyens. L'État n'étant plus le seul «sachant », sa légitimité est plus souvent remise en cause et le rapport de force avec les collectivités s'équilibre.

\subsection{L'avènement de la décentralisation}

Dans les domaines où l'État n'est plus en première ligne, il propose un cadre d'intervention. Il développe fortement dans les années 1990 et 2000 sa politique de prévention des risques littoraux. Il crée de nouveaux outils basés sur une réduction de la vulnérabilité et limite ses participations financières aux projets de défense contre la mer dont les collectivités se sont emparées. Par le développement de dispositifs de contractualisation, il cherche à faire émerger à l'échelle locale des initiatives qui s'inscrivent dans le sillage des stratégies nationales. . C'est le cas de plusieurs dispositifs ouvrant droit à un financement comme les PAPI, détaillés ci-dessous, ou encore de l'appel à projet "Expérimentation de la relocalisation » qui fait suite à l'adoption de la stratégie nationale de gestion intégrée du trait de côte. Les réflexions sur les actions de prévention des risques littoraux sont ainsi de plus en plus partagées. Les commissions départementales des risques naturels majeurs sont des instances de dialogue pour le pilotage de la politique de prévention des risques naturels au niveau départemental. L'État et les collectivités travaillent conjointement sur la mise en ouvre de la directive européenne sur la gestion des inondations, par le biais notamment des stratégiques locales de gestion des risques d'inondation, qui intègrent des principes de gestion intégrée du trait de côte. Leur mise en œuvre est réalisée par le biais de PAPI. Ils reposent sur une gouvernance partenariale rassemblant acteurs locaux et services de l'État, réunis autour d'une vision partagée à la fois du risque inondation sur le territoire et des mesures à prendre localement pour en réduire les conséquences négatives. Plusieurs générations de PAPI se sont succédé $(2002,2011,2018)$. La montée en puissance de cet outil s'accompagne notamment d'une ambition accrue en termes de concertation.

La déclinaison territoriale de ces cadres d'intervention thématiques est cependant confrontée à la logique de territoire. D'un côté, l'État envisage la mise en œuvre d'un outil comme la déclinaison d'une politique publique, pensée de façon sectorielle et selon le principe d'égalité de traitement sur le territoire national. De l'autre côté, la vision des acteurs locaux se fait au travers de leur territoire dans sa globalité et des objectifs qu'ils lui donnent. Ils s'efforcent de les atteindre en appliquant l'ensemble des politiques publiques. Ils suivent donc une approche plurithématique, intégrée sur un territoire restreint. Ce sont donc deux logiques distinctes qui s'affrontent (Figure 3). La spécialisation de l'État au sein de domaines techniques multiples a conduit à une intense production de règles et de normes (Thoenig et Duran, 1996). Leur application sur le territoire a alors amené à identifier des objectifs et des approches difficilement conciliables affaiblissant la légitimité de l'État. La gestion des risques littoraux s'insère ainsi au croisement de deux politiques abordées initialement séparément, la prévention des risques d'inondation et de gestion du trait de côte. Néanmoins, il reste 
difficile pour les collectivités de les dissocier, submersion et recul du trait de côte étant provoqués par les mêmes phénomènes naturels. Les évolutions des rapports entre acteurs, et notamment entre l'État et les collectivités, sont donc très liées à la définition du périmètre du territoire adapté à l'objet de l'action publique. Ainsi, la territorialisation de l'action publique est une des questions majeures posées par la décentralisation.

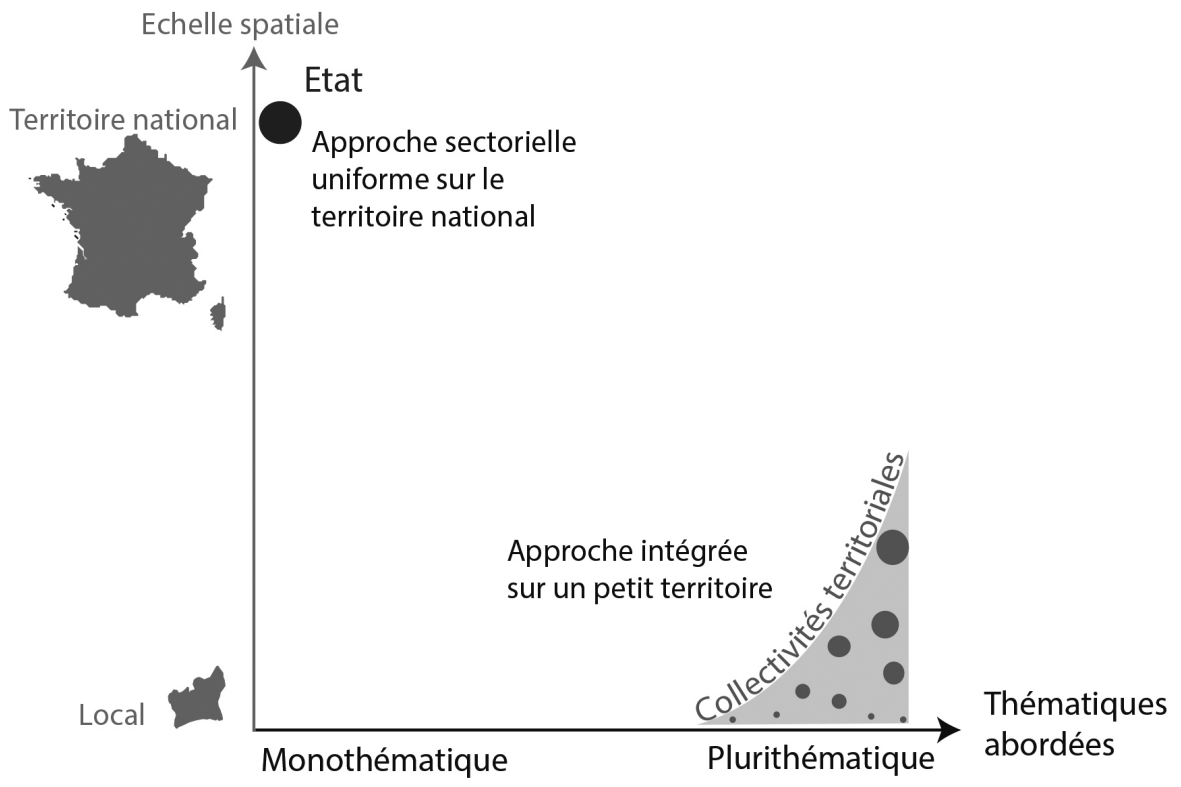

Fig. 3 État et collectivités : deux logiques territoriales et thématiques distinctes National and local government: two different territorial and thematic logical aprroaches

Le domaine des risques littoraux n'a donc pas échappé à la règle et à un système de gouvernance de plus en plus complexe, au fur et à mesure qu'il est partagé (Gaudin, 2002 ; Theys, 2002a). En particulier, la récente prise de compétence GEMAPI bouleverse le rapport à la défense contre la mer des collectivités. La multiplication et l'évolution des outils de prévention des risques complexifient aussi la lisibilité de la politique publique de gestion des risques littoraux. En outre, l'État apparaît déstabilisé par ces évolutions au profit des collectivités territoriales : la conception de la décentralisation n'a pas donné place à l'organisation opérationnelle des nouvelles relations entre l'État et les collectivités. Ces relations se sont façonnées différemment selon chaque territoire (Gaudin, 2004 ; Moquay, 2005). Par ailleurs, les intérêts locaux sont de plus en plus valorisés par rapport à l'intérêt général (Rosanvallon, 2008). Les 
intérêts privés apparaissent encore plus fortement sur le littoral, où la population, majoritairement aisée, dispose de moyens d'actions.

Ainsi, le partage de pouvoir introduit par la décentralisation se fait de manière continue, entre un État héritage d'une tradition jacobine, des collectivités revendicatrices de leurs nouvelles compétences et une population soucieuse de ses intérêts, générant une relation délicate et des incompréhensions dans cette longue période de transition. La décentralisation entraîne la redéfinition d'une nouvelle gouvernance territoriale où tout n'est pas encore écrit.

\section{Des difficultés à surmonter dans les relations entre État et collectivités pour territorialiser la gestion des risques côtiers}

L'historique de la construction de la politique publique de gestion des risques littoraux nous éclaire sur le contexte délicat au sein duquel les acteurs dialoguent aujourd'hui. Les difficultés qu'ils ont à surmonter sont en partie héritées de ce contexte historique, mais aussi de la variabilité des échelles spatiales et temporelles dans lesquelles leurs actions s'inscrivent. Cela est illustré par des extraits d'entretiens réalisés auprès de collectivités (élus et services techniques) et de services de l'État (cf. Tab. 1).

\subsection{Un État encore surplombant}

\subsubsection{Une approche par l'outil et non par le territoire}

Les services de l'État abordent la gestion des risques littoraux avec une entrée thématique alors que les acteurs locaux, et en premier lieu les élus, ont une approche territoriale nécessairement intégrée sur leur territoire (Figure 3), du fait d'une gestion quotidienne d'objectifs, parfois contradictoires, notamment en termes de sécurité et de développement économique de leur commune. Répondre à ces objectifs contradictoires (sécurité vs développement) constitue un dilemme pour les élus locaux (Meur-Ferec et Rabuteau, 2014). L'élaboration d'un PPRL met nettement en évidence cet antagonisme.

Ainsi, les élus reprochent au PPRL d'être coupé du territoire. En effet, l'élaboration de cet outil se focalise sur le risque sans aborder l'aménagement du territoire dans sa globalité (Meschinet de Richemond et Reghezza, 2010). Les acteurs locaux, souvent déjà fortement limités dans les possibilités de développer leur territoire, voient dans les mesures de prévention des risques, une contrainte supplémentaire. "On cumule toutes les..., comme sur beaucoup de côtes j'imagine, beaucoup de contraintes » (élu). «Aujourd'hui entre le PPR, la réglementation liée aux espaces protégés, la réglementation liée aux zones humides, la réglementation au non-étalement urbain, les autres réglementations... Ils ont du mal à trouver des territoires qui peuvent être développés sur leur commune » (service de l'État).

Les élus abordent conjointement l'ensemble des mesures de prévention complémentaires envisageables, là où les services de l'État se focalisent sur un 
seul outil. Ils réclament du «bon sens » en conduisant une réflexion globale. Ils mettent en avant la nécessité de travailler sur d'autres axes de prévention. Ils insistent sur la protection des personnes et la gestion de crise. "Mais on n'a pas protégé la vie humaine. [...] On n'est pas au bout de la démarche » (élu). Ils privilégient très largement les ouvrages de protection à la limitation de l'urbanisation et refusent les hypothèses de défaillance des ouvrages. "La perspective de renforcement des ouvrages de protection ou leur entretien n'est pas pris en compte dans le PPR [...]. Ça, c'est une incompréhension » (élu). Cette réflexion territoriale peut aussi parfois entrainer une volonté de relocaliser certains enjeux, considérés comme trop exposés. «Non seulement il ne faut pas construire, mais il faut évacuer. [...] Je ne comprends pas pourquoi on ne va pas au bout » (élu).

\subsubsection{Des connaissances expertes estimées supérieures aux connaissances territoriales}

La définition des mesures de prévention des risques littoraux s'appuie de façon prépondérante sur des connaissances expertes, techniques, ce qui entrave leur appropriation par les acteurs locaux. En effet, lors de l'élaboration des PPRL, la physique des phénomènes en présence, l'hydraulique et la sédimentologie sont la base de la définition des zones exposées à la submersion marine ou au recul du trait de côte. Les connaissances expertes sont, certes, déclinées localement grâce à des connaissances territoriales, mais les services de l'État affichent rarement de façon explicite l'intérêt des connaissances territoriales dans les études. Ainsi, d'une part ces dernières tendent à être dévalorisées ou du moins sous-exploitées (Martin et al., 2010; Goutx, 2014). D'autre part, l'approche experte qu'empruntent les services de l'État pour présenter ces études est source d'incompréhensions. "C'est extrêmement pointu » (élu). "On ne comprend rien du tout " (élu). Le croisement de ces deux types de savoirs est pourtant un enjeu important, autant pour la qualité des études d'aléas (Ledoux, 2006 ; Mercer et al., 2007) que pour leur appropriation par les acteurs locaux (Perherin, 2017).

La spécialisation des services de l'État et le mode de décision induisent ainsi une asymétrie importante entre l'État et les collectivités et témoignent de la persistance d'un modèle technocratique dans le domaine des risques naturels (Restier-Melleray, 1990 ; Decrop, 2013). Les collectivités territoriales considèrent que l'État impose sa propre vision. "Je pense que ça arrange aussi certains de garder le pouvoir sur certaines connaissances, de pas vulgariser les choses et dans sa grandeur, l'État, dans le cadre du PPRL, l'État a voulu dire: "Nous, on est les sachants. Vous, vous ne comprenez pas et vous allez accepter ce qu'on va vous dire” "(élu). Pour les collectivités, l'État impose, contraint, sans discuter. Leurs formations, leurs connaissances et leur mission de défendre l'intérêt général donnent encore souvent aux services de l'État un sentiment de légitimité dans cette position "surplombante » et la certitude d'agir de manière responsable (Moquay, 2005 ; Gentric et Langumier, 2009 ; Goutx, 2014). Privilégiant le cadre doctrinaire de la prévention des risques naturels et les connaissances expertes à même de définir l'aléa, les services de l'État éprouvent 
souvent des difficultés à dialoguer avec les acteurs qui ont un cadre de pensée différent. Ils ont tendance à redoubler d'explications techniques, les assimilant à un effort de "transparence », face à des acteurs locaux qu'ils estiment être dans la méconnaissance, l'incompréhension ou même l'irresponsabilité (Goutx, 2014).

La méthodologique déployée par l'État pour la mise en place des outils ne conduit ainsi pas à un travail collaboratif, que les collectivités revendiquent autant que leur autonomie.

\subsection{Des collectivités aux revendications discordantes}

\subsubsection{Entre revendications d'autonomie et demandes de soutien}

Il ressort de nos enquêtes que les collectivités cherchent à tirer parti, à la fois des avantages de l'héritage de l'État-providence et de la décentralisation.

Fortes des étapes successives de décentralisation, les collectivités, par la voix de leurs élus revendiquent une plus grande liberté d'action sur « leur » territoire au nom du principe de subsidiarité. Ils critiquent fortement les contraintes d'urbanisation qui leur sont imposées par les lois, notamment la loi Littoral et la loi Barnier. Ils y voient une entrave à leur développement économique (Le Guen, 2004 ; Herviaux et Bizet, 2014). Ainsi par exemple, dans le cadre de projets de relocalisation, les collectivités demandent l'assouplissement de la Loi Littoral. Pour de nombreux élus, elle constitue un obstacle juridique à la réimplantation des enjeux bâtis et donc finalement à la relocalisation : "L'État doit accepter la conversion de zones environnementales en zones constructibles, même si cela doit choquer» (élu). Concernant la loi Barnier, les collectivités vivent souvent comme une ingérence de l'État la faible discussion du zonage réglementaire du PPRL. Elles souhaitent majoritairement pouvoir décider elles-mêmes des zones à aménager, y compris derrière les digues. L'interdiction de construire dans la majorité des zones inondables où la vie n'est pas menacée est également souvent contestée.

Néanmoins, les collectivités sollicitent par ailleurs un soutien financier conséquent de l'État (Le Lidec, 2007). En effet, le fort interventionnisme étatique des décennies précédentes et l'accent mis sur la réparation des dommages via le régime Cat Nat ont encouragé un sentiment de «droit à l'indemnisation ». Selon J. Dubois-Maury (2002) cette situation n'inciterait ni les particuliers ni les collectivités à engager des mesures préventives et à assumer leur responsabilité. Les collectivités se sont également emparées des financements PAPI, notamment pour le renforcement des ouvrages de protection. Elles peuvent également chercher auprès de l'État un appui méthodologique afin de pallier les difficultés qu'elles rencontrent : "S'il y a bien une chose qui est importante c'est que l'État prenne toute la mesure finalement de la complexité de la relocalisation. [...] Il faut que l'État se mobilise avec les collectivités, pas tout seul, mais avec les collectivités pour trouver une méthodologie assez claire » (service technique de collectivité territoriale). Certaines collectivités recherchent ainsi dans l'État un partenaire, parfois un guide, toujours un financeur, particulièrement dans les projets de relocalisation. 
Le rapport qui s'instaure entre les collectivités et l'État, en particulier les attentes des collectivités envers l'État, est fortement influencé par la taille et les moyens des collectivités. Certaines communes, plutôt rurales, disposant de peu de ressources internes et de peu de capacité à conduire des expertises privées, sont plus dépendantes des services de l'État (Bourdieu, 1990): "Les services de l'État pour le maire, c'est la structure qui soutient le maire, qui l'aide dans ses démarches administratives parce que la réglementation est compliquée » (élu). Ailleurs pourtant, les collectivités disposant de plus de ressources financières et politiques mettent en œuvre des moyens importants pour faire contrepoids aux services de l'État (contre-expertises...). « Les petits territoires ruraux, où il n’y a que des élus d'envergure locale, se contentent des arbitrages du préfet. [...] Dès qu'il y a une importance financière ou un poids politique, ça va remonter vite [au cabinet du ministre] » (service de l'État). Dans un système décentralisé, les différences de moyens et de capacité à mobiliser d'autres acteurs, introduisent une iniquité territoriale entre les collectivités.

\subsubsection{Des difficultés à assumer des décisions impopulaires}

En outre, bien que revendiquées par les élus, la décentralisation les place dans une position difficile à tenir à l'échelle locale : celle d'assumer les décisions impopulaires alors qu'ils sont fortement exposés aux sanctions électorales. Cela s'est confirmé dans certains sites de l'appel à projets relocalisation en France, où il n'était plus question d'aborder ce sujet l'année précédant les élections. En effet, les questions d'aménagement constituent des enjeux importants pour les acteurs locaux, et peuvent les mettre en difficulté : "Dans ce contexte [de décentralisation] où l'élu local ne peut plus s'abriter derrière le «tiers absent » que représente l'État, les enjeux d'aménagement deviennent localement des enjeux politiques forts » (Jobert, 1998). En outre, la restriction de l'utilisation de parcelles privées pour des raisons de sécurité ou la relocalisation entraînent des conséquences fortes à titre individuel : « La prévention oblige de prendre des mesures, même impopulaires, dans l'intérêt bien compris de ceux qui n'en veulent pas » (Frémaux, 2002). Les élus subissent des pressions diverses qui peuvent les mettre en difficulté (Meur-Ferec, 2006 ; Beucher et Rode, 2009 ; Douvinet et al., 2011). Ainsi, la contestation des habitants face aux interdictions de construire ou d'investir en zone à risque révèle qu'à l'échelle des particuliers, les intérêts à court terme sont souvent privilégiés : «La population était surtout affectée par sa perte de bien, notamment ceux qui avaient des terrains constructibles qui ne le seront plus » (élu). «Indéniablement, ce n'est pas la sécurité des individus ni des biens, derrière tout ça, c'est l'intérêt financier qui prime » (élu).

Les élus se heurtent également au fait que la population est prête à se mobiliser, à faire appel à des contre-expertises et à la justice pour défendre ses intérêts. "Une des difficultés pour nous c'est quand même le fait que les actes d'urbanisme sont de plus en plus judiciarisés, surtout sur nos territoires où les enjeux environnementaux sont plus forts » (service technique de collectivité 
territoriale). Il est ainsi souvent difficile pour les élus de porter politiquement les mesures de prévention des risques, surtout les projets de relocalisation.

Les élus peuvent alors chercher à se dédouaner des décisions impopulaires. Concernant les projets de relocalisation, ils sollicitent l'État, voire conditionnent leur propre engagement à celui de l'État. Ces attentes vont à contre-courant des processus actuels de décentralisation. Pour mettre en œuvre des décisions impopulaires dont certaines ne se justifient que par l'intérêt général au niveau national, les élus expriment plus ou moins ouvertement le besoin de "se protéger » derrière un tiers : l'autorité de l'État. Ainsi, ils attendent de l'État qu'il assume son rôle d'arbitre et de guide : «La difficulté pour le maire, et je la comprends bien, c'est d'arriver à fixer une limite et là c'est à l'État de se mettre au travail, d'appliquer une limite incontestable : [...] l'interdiction de construire en zone à risque » (service de l'État). L'inaction ou le refus de portage d'un projet est également souvent justifié par l'incertitude scientifique. Comme le note R. Billé (2006) au sujet de projets de gestion intégrée des zones côtières : "On remarque en effet que le manque et la contradiction des connaissances scientifiques sont abondamment utilisés [...] pour retarder des décisions et arbitrages douloureux pour certains acteurs ». La prise en compte de l'élévation du niveau de la mer lié au changement climatique est ainsi souvent rejetée à court terme par les élus, arguant d'un manque de précision et de certitudes dans les prévisions (Perherin, 2017).

Les relations entre l'État et les collectivités se sont d'autant plus complexifiées que des visions très différentes influencent la façon de mettre en œuvre les outils d'application des politiques publiques.

\subsection{La territorialisation : une gouvernance nécessairement partagée et un nouvel équilibre à trouver}

La territorialisation de la politique de gestion des risques côtiers met en exergue des différences de points de vue sur des aspects pourtant fondamentaux. Il s'agit notamment des priorités considérées sur un territoire, de la nature des enjeux à protéger, de la conception de la concertation et de l'échelle de temps dans laquelle les acteurs ancrent leurs logiques.

\subsubsection{Prévention des risques et développement économique : des priorités différentes}

De profondes tensions entre l'État et les collectivités sont liées aux impacts des actions de prévention des risques littoraux sur le développement du territoire (Mineo-Kleiner, 2017 ; Perherin, 2017). En effet, pensée de façon sectorielle, l'application stricte de la doctrine de prévention des risques peut s'opposer aux priorités de développement économique choisies par un territoire comme de "préserver les entreprises et les emplois» (élu). Les élus insistent souvent sur les impacts potentiels du PPRL sur la vie même du territoire. Ils sont nombreux à considérer que le PPRL constitue un "risque » de "mettre fin à toute vie sociale et économique » (élu). Ils rejettent alors ces nouvelles contraintes. "Si, pour sauver un village, il faut le faire crever, alors on a tout faux » (élu). De leur 
côté, les services de l'État retiennent une interprétation basée sur l'objectif de sécurité des biens et des personnes, présentée comme conforme à la doctrine d'élaboration. Ils ont tendance à rester fermes sur leur position et à ignorer les préoccupations de développement économique des territoires, qui restent en dehors de leur champ d'action.

\subsubsection{La prise en compte des dommages matériels : des conceptions différentes des enjeux à protéger}

La conception même des enjeux à protéger conduit à une autre divergence fondamentale de vues entre l'État et les collectivités. Alors que l'État veille à la sécurité des biens et des personnes, les collectivités territoriales se concentrent sur la protection des personnes. En effet, en tant que garant de l'intérêt général et de la pérennisation du système Cat Nat, l'État veille à équilibrer indemnisation et prévention, la prévention des dommages passant par l'élaboration des PPR. Les collectivités, quant à elles, mettent en avant les actions dont elles portent la responsabilité : la sécurité des personnes, liée à leur pouvoir de police et le développement territorial, souvent priorisé semble-t-il. L'objectif de protection des biens n'est que très rarement mentionné par les élus. Bien au contraire, ils expriment souvent une incompréhension par rapport aux contraintes imposées par le PPR sur la constructibilité. Selon les collectivités, les services de l'État ont une approche très « sécuritaire » de la détermination des zones concernées par les PPRL (Vinet et Defossez, 2006 ; Beucher et Rode, 2009 ; Martin et al., 2010). Selon eux, l'État met «ceinture et bretelles» ou sort son «parapluie » (élus). Ce "parapluie ouvert» est interprété comme une protection pour se prémunir de toute mise en cause ultérieure (Carré, 2006 ; Martin et al., 2010). L'incompréhension des élus sur les limitations à la construction est renforcée pour les submersions marines en raison de la prévisibilité des événements météorologiques et des alertes qui permettent une évacuation anticipée des populations. "Sur la submersion marine, la sécurité des gens, c'est l'évacuation » (élu). "Il y a des choses qui s'annoncent avec la météo maintenant. On peut aussi se protéger. Parce l'objectif est de protéger la population. On a les moyens maintenant d'information, compte tenu de notre éloignement par rapport à la côte, de pouvoir mettre à l'abri les habitants » (élu). Ainsi, les élus mettent en avant la nécessité de travailler sur d'autres axes de la prévention que l'interdiction de construire, comme la protection des personnes ou la gestion de crise et l'alerte, et envisagent des relocalisations uniquement dans les rares secteurs qu'ils jugent difficilement évacuables. Dans un contexte de renforcement des aléas côtiers en lien avec le changement climatique, nous pouvons nous interroger sur l'impact de cette faible prise en compte des dommages matériels par les élus locaux qui bénéficient du régime Cat Nat (submersion).

\subsubsection{La concertation comprise différemment selon les acteurs}

Il apparaît que l'État et les collectivités ont des conceptions et donc des attentes bien différentes de la concertation. Ainsi, les services de l'État, utilisent le terme 
de concertation dans un sens générique regroupant différentes formes d'échanges entre acteurs et l'assimilent souvent au terme "consultation ", d'où l'usage récurrent de la curieuse expression "concerter les élus ou la population». Ils ne voient donc pas nécessairement dans la concertation un partage de la décision. Au contraire, les collectivités semblent se référer à une définition "exclusive » du terme concertation, synonyme pour elles de co-construction (Mermet, 2006). Déçues, elles expriment unanimement leur incompréhension face à un processus proche de l'information unilatérale. "On nous a dit: "En concertation avec les élus, voilà ce qu'on a décidé." Alors, on n'a pas la même définition de la concertation " «Non, on a eu de l'information » (élu). "Quand on est en réunion, on n'a pas l'impression d'être concertés, on a l'impression d'être tenus au courant de l'avancée des études » (élu).

Plusieurs raisons expliquent un degré d'ouverture à la concertation limité des services de l'État (Hubert et Reliant, 2003 ; Vinet et Defossez, 2006). Issus de formation principalement technique, sans incitation méthodologique forte ni précise sur le mode de concertation et sans identification des sujets ouverts à la discussion, les agents des services de l'État privilégient une réalisation technique. L'élaboration d'un PPR est d'abord comprise comme un exercice technico-administratif qui n'induit pas pour les agents des services de l'État une réelle fonction d'animation et de concertation. Ils sont également confrontés au délai d'élaboration du PPRL : ils recherchent une certaine efficacité qui pénalise une co-construction gourmande en temps et les incite à privilégier une position "surplombante». Aux contraintes de temps s'ajoutent des contraintes de moyens, financiers et surtout humains (Theys, 2002a ; Hubert et Reliant, 2003 ; Carré, 2006 ; MEDD, 2006) qui pèsent sur les agents et leur laissent une marge de manœuvre limitée dans l'organisation des dispositifs de concertation. Ces conditions pénalisent la réflexion sur l'adéquation des pratiques au contexte et aux exigences d'une gouvernance "moderne » favorisant la coopération..

\subsubsection{Des cadres de pensée inscrits dans différentes échelles temporelles}

Les différences de points de vue entre les acteurs sont aussi fortement liées aux échelles temporelles dans lesquelles ils ancrent leurs actions. Du côté des collectivités, la durée d'un mandat et le devoir de défendre les intérêts des administrés ancrent l'action des maires sur un temps court (Meur-Ferec, 2006). De plus, la gestion quotidienne d'un territoire et ses urgences génèrent de multiples préoccupations qui ne favorisent pas l'émergence de visions à long terme (Meur-Ferec, 2006 ; Lambert, 2015). C'est particulièrement le cas lorsque les moyens humains et financiers disponibles dans la commune sont limités, ou que les maires cumulent plusieurs mandats, ou plusieurs activités. Les évolutions à long terme ne sont pas une préoccupation majeure des collectivités dans la mise en ouvre des politiques locales; elles intègrent peu d'actions de long terme, notamment en termes d'adaptation au changement climatique. Des mandats courts et la proximité avec les citoyens sont difficilement compatibles avec le portage à long terme de politiques de prévention des risques ou de tout 
autre projet s'inscrivant dans une démarche de développement durable : «Sauf incitation extérieure forte, il est difficile d'imaginer que les élus municipaux responsables prennent le risque de s'engager dans des programmes à long terme «altruistes» dont les électeurs auraient à supporter le coût sans en tirer directement les bénéfices» (Theys, 2002b).

Du côté de l'État, les services ministériels n'étant pas soumis aux échéances électorales, ils sont à même de porter une vision sur le long terme. La politique de prévention des risques littoraux, où l'anticipation et le long terme ont une place prépondérante, nécessite ainsi encore un rôle majeur de l'État.

Bien que l'échelle locale soit amenée à jouer un rôle central dans la mise en œuvre des politiques de prévention des risques et de relocalisation, ces freins importants laissent à penser que les élus locaux ne peuvent porter seuls ces politiques. Pour J. Theys (2002b), articuler les actions à court et long terme est essentiel car les projets de développement durable trouveront leur légitimité dans leur capacité à répondre aux attentes locales et aux problèmes immédiats, c'est-à-dire en quelque sorte, à certains intérêts locaux. Comme l'expriment $\mathrm{V}$. Berdoulay et O. Soubeyran (2014), concernant l'adaptation aux changements climatiques, l'intervention de l'État semble nécessaire pour la mise en œuvre du recul anticipé : «Il est clair qu'on est alors tenté de saisir l'adaptation comme un des rares enjeux qui vienne légitimer un retour de la planification par le haut. » Construire une gouvernance multi-niveaux parait alors indispensable (Abel et al. 2011).

\section{Conclusion : une période charnière de pleine reconstruction des équilibres}

Ainsi, la mise en œuvre des politiques de gestion des risques côtiers fait face à un rapport souvent antagoniste entre l'État et les collectivités. Le partage de pouvoir introduit par la décentralisation et dont le processus est toujours en cours, génère une relation délicate, un rapport de force et des incompréhensions. En effet, d'un côté l'État, de tradition jacobine, rencontre des difficultés à se repositionner et à lâcher ses prérogatives (Moquay, 2005). Encore parfois «surplombant", il peine de plus en plus à légitimer son expertise et à faire reconnaitre son rôle de guide. De l'autre, les collectivités sont revendicatrices de leurs nouvelles compétences tout en réclamant plus de moyens financiers à l'État. Il apparait clairement de nos enquêtes que, malgré la décentralisation, la France conserve une tradition d'Étatprovidence fortement centralisé, profondément ancrée dans le fonctionnement de ses institutions et les attentes des citoyens, et marquée par l'indemnisation des catastrophes naturelles fondée sur la solidarité nationale. L'analyse des relations État - collectivités, à la lumière de leur évolution historique, apporte des clés de compréhension de la gestion actuelle des risques côtiers en France.

Ces recherches sur la difficile territorialisation des risques côtiers reflètent finalement un mouvement général de «néolibéralisation » d’une société française 
traditionnellement centralisée et très protégée par l'État qui amène à des injonctions contradictoires : des revendications de liberté locale, mais un maintien des ressources financières issues de la solidarité nationale et une l'assistance méthodologique et technique de l'État, devenue délicate compte tenu de la réduction de ses moyens.

Les évolutions des quarante dernières années en matière d'articulation des politiques et de positionnement des acteurs devraient encore se poursuivre. En effet, la prise de compétence GEMAPI par les collectivités pourrait les inciter à modifier leur stratégie locale de gestion des risques côtiers. Impliqués directement dans le financement des stratégies via la taxe GEMAPI, les riverains participeront peut-être davantage aux débats locaux. Il est probable alors que des réflexions sur l'intérêt général, plus ou moins local (autrement dit les échelles de solidarité), soient rediscutées. En outre, le délicat équilibre observé entre collectivités et services de l'État sera à nouveau certainement ébranlé par la mission parlementaire portant sur la recomposition spatiale des territoires littoraux en vue de leur adaptation au changement climatique (CGEDD, 2019) et le rapport Buchou ${ }^{16}$. Ce dernier devrait aboutir à une proposition de loi pour aborder notamment l'épineuse question du financement de ces recompositions.

Les évolutions de gouvernance sont ainsi au cœur d'un grand chantier, où les intercommunalités sont amenées à jouer un rôle de plus en plus important. Cela débouchera peut-être sur une situation plus saine vis-à-vis des maires qui détiennent des compétences clés pour améliorer la prise en compte des risques dans l'aménagement du territoire, mais qui sont également pris entre leur statut de

16 Rapport de Stéphane Buchou, députée de Vendée, (2019) «Quel littoral pour demain ? vers un nouvel aménagement des territoires côtiers adapté au changement climatique ", rapport remis à M. le Premier ministre et à Madame la Ministre de la Transition Ecologique et Solidaire. https://www.ecologiquesolidaire.gouv.fr/sites/default/files/2019.11.29_Quel-littoral-pour-demain.pdf 
représentant de l'État et leur devoir de défendre les intérêts de leurs administrés. Nous vivons une période charnière de pleine reconstruction de ces équilibres.

\author{
Université de Nantes, Institut de géographie et d'aména- \\ gement \\ Chemin de la Censive-du-Tertre \\ BP 81302 \\ 44312 Nantes Cedex 3 \\ Cerema Risques, eaux et mers \\ 155 rue Pierre-Bouguer \\ BP5 \\ 29280 Plouzané
}

\author{
Université de Bretagne occidentale \\ Institut Universitaire Européen de la Mer \\ Technopôle Brest Iroise \\ Rue Dumont-d'Urville \\ 29280 Plouzané
}

\title{
6 Bibliographie
}

Abel N., Gorddard R., Harman B., Leitch A., Langridge J., Ryan A., et Heyenga S. (2011), "Sea level rise, coastal development et planned retreat: analytical framework, governance principles, an Australian case study », Environmental Science et Policy, vol. 14, n $3: 279-88$. https://doi.org/10.1016/j.envsci.2010.12.002.

André C. (2013), Analyse des dommages liés aux submersions marines et évaluation des coûts induits aux habitations à partir de données d'assurance, Thèse de doctorat, Géographie, Université de Bretagne occidentale, Brest, http://www.nat-hazards-earth-syst-sci.net/13/2003/2013/.

Barraqué B. (1994), "Risque d'inondation : urbanisme réglementaire ou servitude négociée? ", Espaces et Sociétés, vol. 77, n $3: 133$ - 52. https://www.cairn.info/revue-espaces-et-societes1994-3-page-133.htm.

Bayet C. (2000), "Comment mettre le risque en carte? L'évolution de l'articulation entre science et politique dans la cartographie des risques naturels», Politix, vol. 13, n $50: 129-50$. https://doi.org/10.3406/polix.2000.1091.

Berdoulay V. et Soubeyran O. (2014), "Adaptation, science de la durabilité et pensée planificatrice ", Natures Sciences Sociétés, vol. 22, n 2 : 114 - 23. https://doi.org/10.1051/nss/2014024.

Beucher S. et Rode S. (2009), "L'aménagement des territoires face au risque d'inondation : regards croisés sur la Loire Moyenne et le Val-de-Marne», Mappemonde, vol. 94, n $2: 1-19$. http://mappemonde.mgm.fr/num22/articles/art09202.html.

Bezes P. (2009), Réinventer l'État. Les Réformes de l'administration Française (1962-2008). Paris, Presses Universitaires de France. https://doi.org/10.3917/puf.bezes.2009.01.

Billé R. (2006), "Gestion intégrée des zones côtières : quatre illusions bien ancrées ", VertigO - La revue électronique en sciences de l'environnement, vol. 7, n $3: 1$ - 22. https://doi.org/10.4000/ vertigo.1555. 
Bourdieu P. (1990), « Droit et passe-droit : le champ des pouvoirs territoriaux et la mise en œuvre des règlements ", Actes de La recherche en sciences sociales, vol. 81 - 82 : 86 - 96. https://doi.org/ 10.3406/arss.1990.2928.

Callon M., Barthe Y. et Lascoumes P. (2001), Agir dans un monde incertain. Essai sur la démocratie technique, Paris, Le Seuil, p. 448 p.

Carré C. (2006), «Les évolutions en France dans la théorie et les pratiques d'une gestion territoriale du risque : I'application au cas des inondations », Annales de Géographie, vol. 2, n 648 : 133 53. https://doi.org/10.3917/ag.648.0133.

Cazaux E., Meur-Férec C. et Peinturier C. (2019), "Le régime d'assurance des catastrophes naturelles à l'épreuve des risques côtiers. Aléas versus aménités, le cas particulier des territoires littoraux ", Cybergeo : European Journal of Geography, Espace, Société, Territoire, document 898. https://doi. org/10.4000/cybergeo.32249.

Conseil Général de l'Environnement et du Développement durable (CGEDD) (2019), Recomposition spatiale des territoires littoraux. Rapport au gouvernement.

Deboudt P. (2010), « Vers la mise en œuvre d'une action collective pour gérer les risques littoraux en France métropolitaine », Cybergeo : European Journal of Geography. Espace, société, territoire, $\mathrm{n}^{\circ} 491$ (mars). https://doi.org/10.4000/cybergeo.22964.

Decrop G. (2013), «Expertise des risques et risques de l'expertise », Troisièmes Journées « Aléa gravitaire », Grenoble, France.

Douvinet J., Defossez S., Anselle A. et Denolle A.-S. (2011), « Les maires face aux plans de prévention du risque inondation (PPRI) », L'Espace Géographique, vol. 40, n 1 : 31-46. http://www.cairn. info/revue-espace-geographique-2011-1-page-31.htm.

Dubois-Maury J. (2002), « les risques naturels en France, entre réglementation spatiale et solidarité de I'indemnisation ", Annales de Géographie, n 627/628 : 637-51.

Ewald F. (1986), L'État Providence, Paris, Grasset, p. 610 p.

Frémaux C. (2002), "La responsabilité des maires face aux risques naturels », Annales des Mines: 43-48. http://www.annales.org/ri/2002/temp-08-02.html.

Gaudin J.-P. (2002), Pourquoi La Gouvernance?, Paris, Presses de Sciences Po, La Bibliothèque du Citoyen, p. 140 p.

Gaudin J.-P. (2004), «XVII. La contractualisation des rapports entre l'État et les collectivités territoriales », Annuaire des collectivités locales, n 24 : 215 - 34. https://doi.org/10.3406/coloc.2004. 1540.

Gentric J. et Langumier J. (2009), "Inondations des villes, inondations des champs. » Natures Sciences Sociétés, vol. $17, \mathrm{n}^{\circ} 3: 257-65$.

Goutx D. (2014), « Les leçons de l'incorporation de l'expertise hydrogéomorphologique dans la doctrine française de prévention des risques d'inondation », Vertigo - la revue électronique en sciences de l'environnement, vol. 14, n² 2 . http://vertigo.revues.org/15036.

Habermas J. (1973), La technique et la science comme "idéologie », Paris, Gallimard, 262 p.

Herviaux O. et Bizet J. (2014), "Plaidoyer pour une décentralisation de la Loi "Littoral" : un retour aux origines » https://www.senat.fr/rap/r13-297/r13-297.html.

Hubert G. et Reliant C. (2003), «Cartographie réglementaire du risque d'inondation : décision autoritaire ou négociée? ", Annales des Ponts et Chaussées, n 105 : 24-31.

Jobert A. (1998), « L'aménagement en politique. Ou ce que le syndrome NIMBY nous dit de l'intérêt général », Politix, vol. 11, nº 42 : 67 - 92. https://doi.org/10.3406/polix.1998.1725.

Lambert M.-L. (2015), "Le recul stratégique : de l'anticipation nécessaire aux innovations juridiques », Vertigo - la revue électronique en sciences de l'environnement, vol. 21 (avril). https://doi.org/10. 4000/vertigo.15812. 
Lascoumes P. (2002), " L'expertise, de la recherche d'une action rationnelle à la démocratisation des connaissances et des choix », Revue Française d'Administration Publique, vol. 103, n 3 : 369. https://doi.org/10.3917/rfap.103.0369.

Le Bourhis J.-P. (2007), « Du savoir cartographique au pouvoir bureaucratique. les cartes des zones inondables dans la politique des risques (1970-2000) », Genèses, vol. 3, n68 : 75 - 96. http://www. cairn.info/revue-geneses-2007-3-page-75.htm.

Le Galès P. (1995), "Du gouvernement des villes à la gouvernance urbaine », Revue Française de Science Politique, vol. 45, $\mathrm{n}^{\circ} 2$ : 57-95.

Le Guen J. (2004), « Rapport d'information de I'Assemblée Nationale sur la Loi Littoral » http://www. assemblee-nationale.fr/12/pdf/rap-info/i1740.pdf.

Le Lidec P. (2007), « Le jeu du compromis : I'État et les collectivités territoriales dans la décentralisation en France ", Revue Française d'Administration Publique, vol. 1, n 121 - 122 : 111 - 30. https://doi. org/10.3917/rfap.121.0111.

Ledoux B. (2006), La Gestion du risque inondation, Paris, T. Lavoisier, 766 p.

Martin B., Ansel R. et Guerrouah O. (2010), "Territorialisation ou déterritorialisation du risque? analyse comparative et critique de la procédure de réalisation des PPRNP » Riseo : Risques Études et Observations, $\mathrm{n}^{\circ} 1$ : 83-98. http://www.riseo.fr/IMG/pdf/Riseo_2010-1_Observations_6.pdf.

MATE (Ministère de I'Aménagement du Territoire et de I'Environnement), et METL (Ministère de l'Équipement des Transports et du Logement), (1997), Plans de prévention des risques naturels $(P P R)$. Guide général. La Documentation Française.

Mazé C. et Meur-Ferec C. (2017), «Littoral ». In N. Kada, R. Pasquier, C. Courtecuisse, V. Aubelle (dir.), Dictionnaire encyclopédique de la décentralisation, Berger-Levrault, Hors collection, 1096 p.

MEDD (Ministère de l'Écologie et du Développement durable) (2003), Plans de Prévention des Risques Naturels (PPR). Guide de la concertation. La Documentation française, 62 p.

MEDD (Ministère de l'Écologie et du Développement durable) (2006), Information, participation du public, concertation et association dans les plans de prévention des risques, Direction de la prévention des pollutions et des risques, CERTU.

MEEM (Ministère de I'Environnement, de I'Énergie et de la Mer), MLHD (ministère du Logement et de l'Habitat durable) (2016), Plans de prévention des risques naturels prévisibles. Guide général. Direction générale de la prévention des risques, Direction générale de l'aménagement, du logement et de la nature, $179 \mathrm{p}$.

Mercer J., Dominey-Howes D., Kelman I. et Lloyd K. (2007), " the potential for combining indigenous et western knowledge in reducing vulnerability to environmental hazards in small island developing states », Environmental Hazards, Vol. 7, nº $4: 245-256$.

Merckelbagh A. (2009), Et si le littoral allait jusqu'à la mer! la politique du littoral sous la $V^{e}$ République. Versailles, Quae, 352 p.

Mermet L. (2006), « La « concertation » : un terme flottant pour un domaine mouvant ? » Négociations, vol. 5, $\mathrm{n}^{\circ} 1$ : 75. https://doi.org/10.3917/neg.005.0075.

Meschinet de Richemond N. et Reghezza M. (2010), "La gestion du risque en France : contre ou avec le territoire? ", Annales de géographie, vol. 673, n $3: 248-267$. https ://www.cairn.info/revueannales-de-geographie-2010-3-page-248.htm

Meur-Ferec C. (2006), «De la dynamique naturelle à la gestion intégrée de l'espace littoral : un itinéraire de géographe », habilitation à diriger des recherches, Géographie, Université de Bretagne occidentale, Brest, 247 p.

Meur-Ferec C., Lageat Y. et Hénaff A. (2013), « La gestion des risques côtiers en France métropolitaine : évolution des doctrines, inertie des pratiques ?", Géorisques, n 4 : 57-67. http://hal.univ-nantes. fr/hal-00430767/. 
Meur-Ferec C. et Morel V. (2004), "L'érosion sur la frange côtière : un exemple de gestion des risques ", Natures Sciences et Sociétés, vol. 3, nº 12 : 263-273. https://doi.org/10.1051/nss:2004038.

Meur-Ferec C. et Rabuteau Y. (2014), «Plonevez-les-Flots : un territoire fictif pour souligner les dilemmes des élus locaux face à la gestion des risques côtiers », L'Espace Géographique, Tome 43, n¹ : 18 - 34. http://www.cairn.info/article.php?ID_ARTICLE=EG_431_0018.

Mineo-Kleiner L. (2017), L'option de la relocalisation des activités et des biens face aux risques côtiers : stratégies et enjeux territoriaux en France et au Québec, Thèse de doctorat, Géographie, Université de Bretagne occidentale, Brest, https://tel.archives-ouvertes.fr/tel-01611685.

Moquay P. (2005), «L'État territorialisé ou l'arroseur arrosé : les ambiguïtés de l'État face aux recompositions territoriales en France. » In Bherer L., Collin J.-P., Kerrouche E., et Palard J. (dir.), Jeux d'échelle et transformation de l'État : le gouvernement des territoires au Québec et en France, Laval, Les presses de l'Université Laval : 85 - 116.

Paoletti M. (2007), Décentraliser d'accord, démocratiser d'abord. Le gouvernement local en question, Paris, La Découverte, 154 p.

Peretti-Watel P. (2000), Sociologie du risque. Paris, A. Colin, Collection U : Série Sociologie.

Perherin C. (2017), La concertation lors de la cartographie des aléas littoraux dans les plans de prévention des risques : enjeu majeur de prévention, Thèse de doctorat, Géographie, Université de Bretagne occidentale, Brest. https://tel.archives-ouvertes.fr/tel-01722799.

Restier-Melleray C. (1990), "Experts et expertise scientifique. Le cas de la France », Revue Française de Science Politique, vol. 40, n 4 : 546-585. https://doi.org/10.3406/rfsp.1990.394498.

Rosanvallon P. (1990), L'État en France de 1789 à nos jours, Paris, Le Seuil, 384 p.

Rosanvallon P. (2008), La légitimité démocratique. Impartialité, réflexivité, proximité, Paris, Le Seuil, $384 \mathrm{p}$.

Theys J. (2002a), " La gouvernance, entre innovation et impuissance », Développement Durable et Territoires, vol. 2. https://doi.org/10.4000/developpementdurable.1523.

Theys J. (2002b), " l'approche territoriale du " développement durable », condition d'une prise en compte de sa dimension sociale », Développement Durable et Territoires, $\mathrm{n}^{\circ} 1: 1-12$. https://doi.org/10.4000/developpementdurable.1475.

Thoenig J.-C. et Duran P. (1996), "L'État et la gestion publique territoriale », Revue Française de Science Politique, vol. 46, $\mathrm{n}^{\circ} 4$ : 580- 623. https://doi.org/10.3406/rfsp.1996.395082.

Vinet F. et Defossez S. (2006), "La représentation du risque d'inondation et de sa prévention », In Laganier R. (dir.), Territoires, inondation et figures du risque. La prévention au prisme de l'évaluation, Paris, L'Harmattan : 99-137. 